\title{
A rare complex mutation in FMF gene; genetics and treatment decisions in Familial Mediterranean Fever patients
} Introduction: FMF is an autosomal-recessive autoinflammatory disease characterized by episodic, self-
limiting attacks characterized by fever, abdominal pain, pleurisy, arthritis and erysipelas-like-erythema.
Systemic amyloidosis is the most severe manifestation of the disease.

Case: 3 year old patient for evaluation of FMF who was referred because of gastrointestinal symptoms and a paternal family history of FMF. He had a number of gastrointestinal symptoms as an infant up to the age of 8 months, since the age of 1 year the patient had had 2 episodes each lasting 2 months, during which he would be sick every week with fever and severe abdominal pain and vomiting. The patient met the criteria for FMF based on the criteria from the Sheba medical center of Tel Hashomer in Israel. The patient was determined to have rare heterozygous complex mutation with E148Q-P369S-R408Q in cis state. Testing of his parents revealed that he had inherited all of the mutations from his mother despite the paternal familial history of FMF. Since amyloidosis is rare in Ashkenazi families and due to absence of mutations liked with high risk of amyloidosis, it was determined that he did not need chronic colchicine therapy. To best of our knowledge this is the third reported case of FMF with this gene complex and the first reported case in a patient of Shephardic and Ashkenazi Jewish ancestry.

Conclusion: E148Q, P369S and R408Q when present separately are associated with a mild phenotype and incomplete penetrance, but when present together in cis form on same allele may lead to increased penetrance and clinical disease.

Keywords: FMF gene mutation • E148Q-P369S-R408Q FMF gene mutation • auto inflammatory diseases - amyloidosis in FMF. familial mediterranean fever

\section{Introduction}

FMF is an autosomal-recessive autoinflammatory disease characterized by episodic, self-limiting attacks characterized by fever, abdominal pain, pleurisy, arthritis and erysipelas-like-erythema. Most patients remain free from fever and inflammation in between the episodes. Systemic amyloidosis is the most severe manifestation of the disease. It results from amyloid protein-A deposition, commonly affecting the kidneys and sometimes adrenal glands, intestine, spleen, lungs and testis [1]. Ethnicity appears to contribute to the risk of developing amyloidosis, with reported higher frequency in Sephardic Jews of North African ancestry, particularly in comparison to Ashkenazi Jews [2]. The ability to use genetic testing recently allowed us to make a determination whether prophylactic versus intermittent colchicine was most appropriate for a patient with a Sephardic father and Ashkenazi mother.

\section{Case}

We recently saw a 3 year old patient for evaluation of FMF who was referred because of gastrointestinal symptoms and a paternal family history of FMF. He had a number of gastrointestinal symptoms as an infant up to the age of 8 months. Over following 2 years, the patient had 2 episodes each lasting for 2 months during which he would be sick every week with fever and severe abdominal pain and vomiting lasting 2-3 days. In between the episodes patient would be symptom free. The patient met the criteria for FMF based on the criteria from the Sheba medical center of Tel Hashomer in Israel [3]. He had 1 minor criterion (we considered the attacks incomplete attacks as patient was not examined by us during the typical attack) and 6 supportive criteria:
- family history of FMF

\section{Shailendra Singh ${ }^{* 1}$, Jyoti Chaudhary $^{2}$ \& John Meyerhoff ${ }^{3}$ 'Medical Director of Rheumatology, White River Medical Canter, USA \\ ${ }^{2}$ Department of Rheumatology and Internal Medicine, Whitye River Medical Center, USA \\ ${ }^{3}$ Johns Hopkins University and Sinai Hospital of Baltimore, USA}

*Author for correspondence: shailendra231@gmail.com 
- appropriate ethnic origin

- age $<20$ years at onset

- severe attacks requiring bed rest

- spontaneous remission, and

- symptom free interval

Due to the Sephardic father's family history of FMF there was a concern that the patient could be at increased risk of amyloidosis if he had inherited the disease from his father. Therefore it was decided to do genetic testing to see if the patient had any MEFV mutations associated with FMF and a possible increased risk of amyloidosis.

Buccal swab mucosa was sent to GeneDx to evaluate for MEFV gene mutation. DNA was PCR-amplified for analysis of exon 2, 3 and 10 of the MEFV gene, bi-directional sequence was obtained and DNA sequence was analyzed and compared to the published gene sequence.

The patient was determined to have heterozygous mutations of E148Q, P369S, and R408Q. The presence of this mutation is supportive of the diagnosis of FMF. This further raised the question whether the patient inherited these mutations from one parent (cis) or from both (trans). Also as the patient had a mutation in only one gene it became very important to determine if he had received this gene from his Sephardic father or from his Ashkenazi mother. Although these particular mutations not have been associated with amyloidosis, there is a risk of amyloidosis even if a patient does not have the usual amyloidosis gene. The risk of amyloidosis is usually much higher in the Sephardic population then the Ashkenazi population. So we felt it was important to determine which parent the patient has inherited this gene from. After detailed discussion with the parents, it was decided to do gene testing in both the parents to determine which parent is contributing the genes for FMF.

The patient's father tested negative for all the mutations noted in the child while his mother was positive for all three mutations; therefore the patient inherited all the mutations from his mother. Therefore his risk of amyloidosis was felt to be extremely low due to his lack of the mutations reported to be associated with amyloidosis and the Ashkenazi background of his mother.

As the patient had had only two episodes of attacks over 2 years, after discussion with the parents it was decided that we would follow patient and use intermittent colchicine therapy during the attacks to help with symptoms [4]. Over last 2 years (age 3-5 years) patient has not any more episodes suggestive of FMF. If he has been on daily colchicine the lack of symptoms would have been attributed to the colchicine and the patient would likely to have been continued on it for many years, if not his lifetime.

\section{Discussion}

On an extensive review of the literature we found only two reported cases of FMF with heterozygous complex mutation with E148Q-P369S-R408Q in cis state with classic FMF symptoms. An Armenian patient had classical FMF with fever and peritonitis but no documented arthritis or amyloidosis [5]. A second case was reported in a Japanese patient but the clinical features were not reported [6].

Therefore our patient may be the third reported case of FMF with this gene complex and the first reported case in a patient of Shephardic and Ashkenazi Jewish ancestry.

A review of MEFV testing at the NIAMS found 5 patients with only the P369S and R408Q mutations who met the criteria for FMF by Livneh. These 5 patients were defined as having "incomplete" attacks because, like our patient, they were not seen during the attacks. Six additional patients with these two mutations and a single E148Q mutation and one who was homozygous for E148Q were reported as having unusual sets of symptoms not meeting criteria. There may be a larger group of FMF patients with "atypical" manifestations. Grateau et al. reported 22 patients with two mutations in exon 10 (homozygotes and compound heterozygotes) with "atypical" features. Like the NIAMS series, since many of the patients were tested but not seen by the authors, complete clinical data is not available. None of the 22 was reported to have responded to colchicine, but this includes nonresponders as well as those who were not treated either.

The most perplexing questions in the genetics of FMF are 1) what is the significance of mutations outside of exon 10 and 2) how do patients with heterozygous mutations develop symptoms of a disease that is felt to be an autosomal recessive disease.

The M680I, M694I, M694V and V726A mutations on exon 10 are the most common and are generally associated with more severe 
disease when present in homozygous state. Along with the E148Q mutation on exon 2, they may account for the mutations seen in approximately $80 \%$ of classically affected FMF patients [1].

There have been discordant results in studies looking for an amyloidosis mutation in FMF patients. The failure of some investigators to find the same result may be due to low penetrance of the gene in some or all of the patients. This may explain why the one group which reported an increase in M694V frequency among 29 patients with FMF with amyloidosis found the M694V mutation in 27 homozygotes.

The significance of the E148Q mutation is debated. It has been reported to occur on $24 \%$ of FMF chromosomes that do not have a mutation on exon [7-10]. It has been reported in patients from many different ethnic backgrounds but more commonly in Ashkenazi Jews. Patients with only this mutation tend to have milder disease with less arthritis, more pleuritis and almost no amyloidosis. Fifty percent of E148Q homozygotes are asymptomatic, suggesting low penetrance, and expression only under certain circumstances, genetic backgrounds or environmental factors [7]. However when E148Q is part of the complex allele involving mutations in exon 10, it has been associated with severe disease and complications. The most commonly reported complex mutations are E148Q-V726A (gene frequency 5.1\%) in Ashkenazi Jews and E148Q-M694P in North African Jews [7,8].

The P369S is mutation located on Exon 3 of MEFV gene and occurs in 2-3\% of MEFV mutations carrying chromosomes. This mutation had been described predominantly in individuals of Armenian and Ashkenazi Jewish ancestry. Just like E148Q it has been associated with a mild phenotype and incomplete penetrance [9]. R408Q has been described rarely and nearly always in cis phase with P369S. Recent studies have reported P369S and R408Q being in strong linkage disequilibrium in Japanese population, suggesting they cosegregate in cis form. P369S/ R408Q substitutions are associated with highly variable phenotypes and are infrequently associated with typical FMF symptoms. Both substitutions P369S and R408Q are on Exon 3 which encodes pyrin's B-box domain, necessary for interaction of Pyrin with PSTPIP1. However no significant correlations had been demonstrated in the mutation and effect on pyrin's binding to PSTPIP1 [10].
FMF has been classically transmitted as an autosomal recessive trait, however, it had been observed that a substantial number of patients possessing only one demonstrable mutation despite sequencing the entire mutation. Three British families have been identified with probably true dominant FMF [11] as appears to be the case with a large family of Americans of Lebenese previously reported [12]. This appears to be the case with our patient, but then the question arises why the mother has no symptoms.

There have been reported cases in literature of late onset FMF especially in women with heterozygote mutations; these cases of late onset FMF may represent a "non-penetrant" female segment of the FMF population in which the symptoms only manifest with the withdrawal of the ameliorating effect of estrogens. Perhaps, as our patient's mother goes through menopause she may develop symptoms of FMF. This may explain why our patient, a male, has symptoms, but his mother does not.

The absence of FMF amyloidosis associated mutations and determination that this patients possible FMF was from his Ashkenazi Jew mother allowed us the opportunity to avoid chronic prophylactic colchicines and observe the natural course of his symptoms.

\section{References}

1. Booty MG. Familial Mediterranean fever with a single MEFV mutation: where is the second hit? Arthritis. Rheum. 60(6), 1851-61 (2009).

2. Aksentijevich I. Familial mediterranean fever at the Millennium Clinical spectrum, Ancient Mutations and Survey of 100 American referrals to National institute of health. Medicine (Baltimore). 77(4), 268-97 (1998).

3. Livneh A. Criteria for the diagnosis of familial Mediterranean fever. Arthritis. Rheum. 40(10), 1879-85 (1997).

4. Wright DG. Efficacy of intermittent colchicine therapy in familial Mediterranean fever. Ann. Intern. Med. 86(2), 162-5 (1977)

5. Cazeneuve C, Sarkisian T, Pêcheuxet T et al. MEFVGene Analysis in Armenian Patients with Familial Mediterranean Fever: Diagnostic Value and Unfavorable Renal Prognosis of the M694V ic and Therapeutic Implications; Am. J. Hum. Genet. 65, 88-97 (1999).

6. Tsuchiya-Suzuki A. Clinical and genetic features of familial Mediterranean fever in Japan. J. Rheumatol. 36(8), 1671-6 (2009).

7. Ben-Chetrit E. Familial Mediterranean Fever in the world. Arthritis. Rheum. 61(10), 1447-53 (2009).

8. Gershoni-Baruch R. The differential contribution of MEFV mutant alleles to the clinical profile of familial Mediterranean fever. Eur. J. Hum. Genet. 10(2), 145-9 (2002). 


\section{Case Report Singh, Chaudhary, Meyerhoff}

9. Touitou I. The spectrum of Familial Mediterranean Fever (FMF) mutations. Eur. J. Hum. Genet. 9(7), 47383 (2001).

10. Ryan JG. Clinical features and functional significance of the P369S/R408Q variant in pyrin, the familial Mediterranean fever protein. Hum. Immunol. 70(6), 468-71 (2009)
11. Booth DR, Gillmore JD, Lachmann HJ et al. The genetic basis of autosomal dominant familial Mediterranean fever. QJM: An international Journal of Medicine. 93(4), 217-21 (2000).

12. Meyerhoff J. Familial Mediterranean fever: report of a large family, review of the literature, and discussion of the frequency of amyloidosis. Medicine (Baltimore). 59(1), 66-77 (1980) 